\title{
A first approximation of population distributions on the
}

\section{International Space Station}

\author{
Justin St. P. Walsh ${ }^{1}$ and Rao Hamza Ali ${ }^{2}$ \\ Chapman University, Orange, CA 92866
}

\author{
Alice C. Gorman ${ }^{3}$ and Amir Kanan Kashefi ${ }^{4}$ \\ Flinders University, Adelaide, South Australia 5042, Australia
}

This article presents an analysis of data derived from thousands of publicly-available photographs showing life on the International Space Station (ISS) between 2000 and 2020 . The analysis uses crew and locational information from the photographs to identify the distribution of different population groups - by gender, nationality, and space agency affiliation - across modules of ISS for the first time. Given the importance of ISS as the most intensively-inhabited space habitat to date, an international cooperative initiative involving 26 countries and five space agencies, and one of the most expensive building projects ever undertaken by humans, developing an understanding of which people are using different parts of the space station is critical for future usage of this and other stations. This study also sheds light on problems faced by future space station designers who are concerned with optimal usage of their habitats.

\footnotetext{
${ }^{1}$ Associate professor of art history and archaeology, Department of Art (corresponding author).

2 Doctoral candidate, Schmid College of Science and Technology.

${ }^{3}$ Associate professor of archaeology, College of Humanities, Arts, and Social Sciences.

${ }^{4}$ Database engineer, College of Humanities, Arts, and Social Sciences.
} 


\section{ISS Configuration}

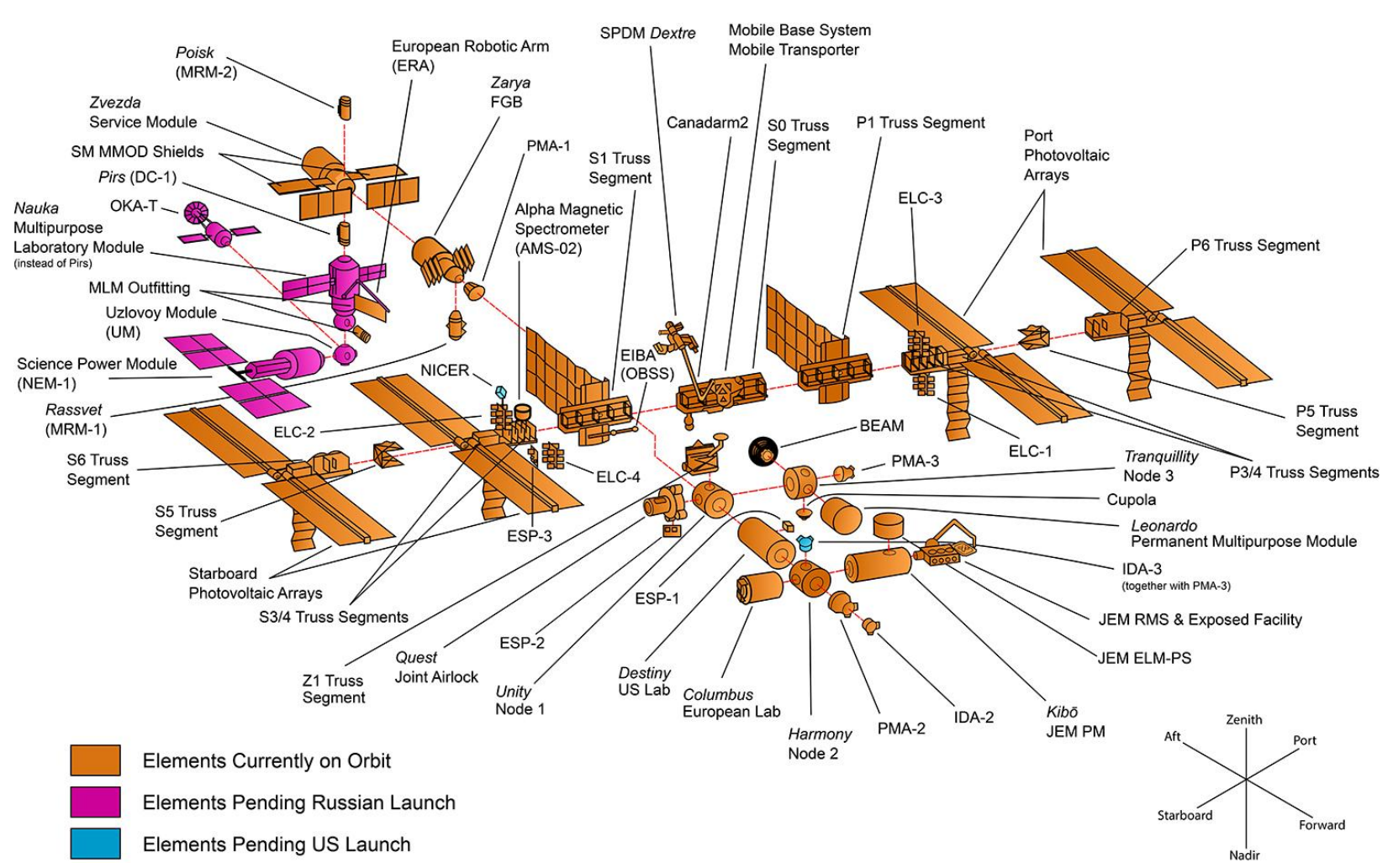

Fig. 1. Exploded view of the International Space Station, showing its constituent modules. (NASA, by permission)

\section{Introduction}

The International Space Station (fig. 1) has been continuously inhabited for more than twenty-one years. In that time, it has been visited by more than 245 individuals from nineteen countries and ten space agencies. It is thus the longest- and most intensively-occupied space habitat launched so far. In this study, we ask a simple question about the occupation of the space station: who is using the different parts of the space station? To put it another way, more specifically: what are the distribution patterns of males and females, people of different nationalities, and members of different space agencies across various modules of ISS? These questions are highly significant because space agencies and the commercial space industry are planning new stations and even more intensive missions (for example, to Mars) with 
durations that go far beyond the average of 238 days served by ISS's long-term crew (this average was calculated for the 113 crew members who served on ISS for more than 48 total days through Expedition 63 (mid-2020)) (fig. 2).

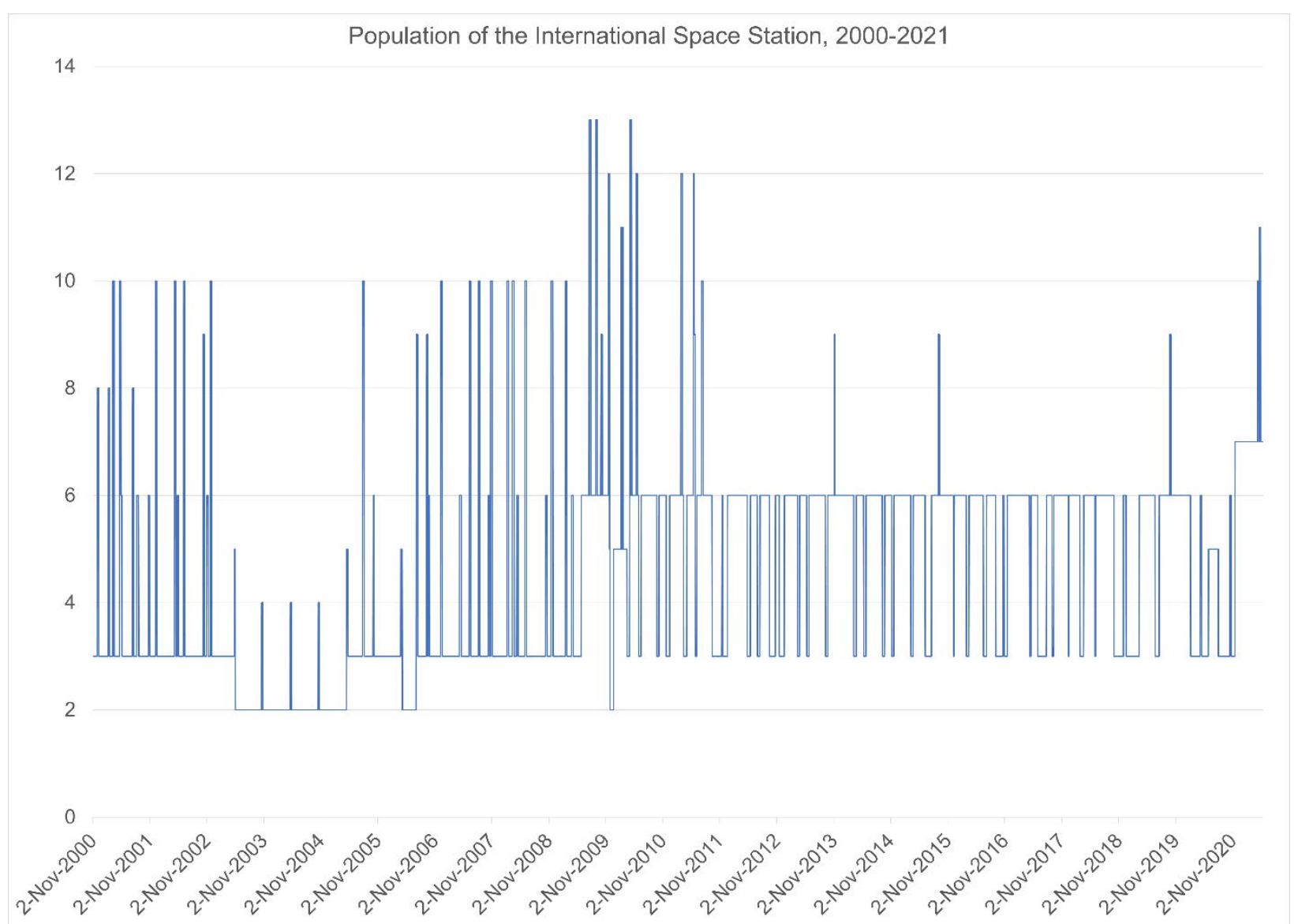

Fig. 2. Population of the International Space Station between November 2000 and May 2021.

Understanding which individuals are using which areas of any habitat, let alone a habitat costing many tens of billions of dollars to build and maintain, is critically important for improving both future designs for architecture and work plans to be carried out by crew. Given the amount of attention paid by space agencies to the daily activities of their crew, these questions seem superficially like they should have straightforward answers. After all, astronauts are continually monitored by video in real time during their working hours (and possibly at other times as well), crew schedules are organized in increments as small as 5 minutes in the official ISS Operations Planner [1,2,3], and there are extensive public records of who was present on the space station at any moment. But no analysis of population distributions across ISS 
modules has been done, and, indeed, the raw data needed to perform such an analysis has never been generated from the existing evidence in agency archives. The evidence is, admittedly, difficult to work with. For example, crew work schedules are unreliable sources on their own, since they are subject to frequent changes: projects are rearranged due to unforeseen contingencies, crew are often interrupted during specified tasks, and sometimes they have to work in multiple locations simultaneously. These schedules also do not account for people's locations in periods outside of working hours.

\section{Data}

For this study, we have been collecting occupation data and analyzing it for significant patterns [4]. Here, we report results from Phase 1 of this work, using the metadata from thousands of historic photographs as evidence, together with a list of crew members and their genders, nationalities, and agency affiliations. The images were collected by NASA on behalf of four of the five international partner agencies that run ISS. The photos held by NASA represent imagery made by crew from that agency as well as from the Japanese Aerospace Exploration Agency (JAXA), the European Space Agency (ESA), and the Canadian Space Agency (CSA). The Russian agency, the Roscosmos State Corporation for Space Activities, maintains its own photo archive through its spacecraft contractor, PAO S. P. Korolev Rocket and Space Corporation Energia. We have so far been unable to access this archive (see below for a discussion of the impact created by this lacuna).

Digital photography was adopted by the agencies from the beginning of the space station program, allowing for many more images of ISS to be recorded than of previous space missions. By observing these images, we can identify the crew members and locations depicted in them. Work with the photos has required several stages of development due to the large number of images, as well as the complexity of extracting usable data from them. The images analyzed in this article were published by NASA's Public Affairs Office on its page on Flickr, the image-hosting website [5]. There are 8,291 interior images from the first 63 mission increments, or Expeditions, in the Flickr set, covering the years 2000-2020. NASA maintains a much larger set of ISS photos for its own internal use. With the award of 
an Australian Research Council Discovery Program grant in 2019, we were able to gain access to the larger photo set; the results from analysis of that larger set will be the subject of a future article (see below).

\section{Method}

Initial testing using the Flickr photo set showed that manual tagging of people, places, and items could take hours per photo [4]. At the same time, our approach was validated by a pilot study of items displayed on the aft wall of the Russian Zvezda module [6,7]. In that work, we were able to begin to define cultural associations of specific religious and secular items with groups of cosmonauts and a particular location. We documented 414 instances of 78 unique items (including Russian Orthodox painted icons and portraits of space heroes such as Yuri Gagarin) shown in 48 photos dating between 2000 and 2014. It became clear from the initial work, though, that we needed to find a way to automate the tagging of items in the photos.

We identified two ways of accelerating the process that rely on information technology: developing a crowdsourcing platform that allows "community scientists" to contribute tags based on recognizable features in the images [8]; or harnessing the power of artificial intelligence (AI) software to automate the tagging process [9]. Each approach presents its own advantages and difficulties.

Crowdsourcing allows for public science outreach simultaneous with research activity, but it also requires the creation of a website with tutorials, high-quality visual design, and workflows for vetting data quality [10]. Crowdsourced tagging further becomes more complex as the task at hand caters to relatively specific data and tagging requirements [11]. By contrast, AI software running on powerful computers can tag hundreds of photos per second, but for specialized photo sets such as the ISS images, a significant investment in time and effort is required to pre-train the algorithms to recognize the kinds of things that will appear in the pictures [12]. A hybridized combination of the two approaches, where AI is used to evaluate the crowdsourced tags, has been applied in other studies, but there too the inefficiency of human tagging on complex data is highlighted [13]. We chose to use AI-based machine learning (ML) algorithms 
to perform the primary tagging of the larger photo set. This choice was necessitated by restrictions placed on the distribution of previously-unpublished photos by NASA; some of the images cannot be released because they show proprietary commercial equipment, while others could infringe on the privacy of crew. These images cannot be published or made publicly available, e.g., to a group of community science participants.

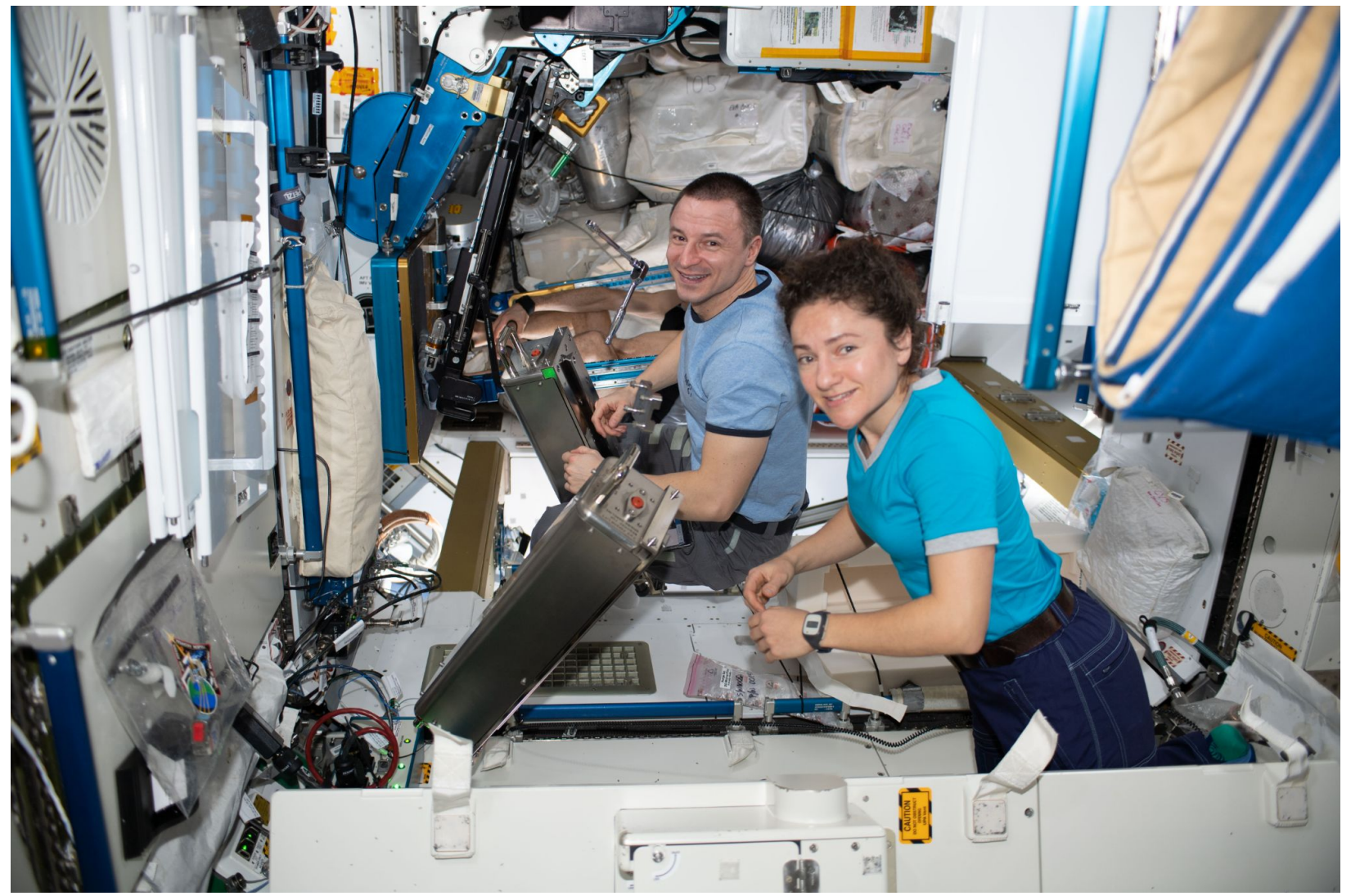

Fig. 3. A typical photograph published by NASA on its Flickr site, showing two crew members performing maintenance. (NASA, by permission) 
We were able to capture the identities of the people and places in these images using the natural text descriptions. We automated the extraction of relevant content, using the Flickr API and web scraping software developed by our team [15]. This software identifies, saves, and processes information from the web. For this project, the scraper accesses the Flickr site, parses the content of each page, finds the data of interest (in the example above, "Jessica Meir," “Andrew Morgan,” and “Tranquility”), and finally structures the data as needed. The scraping also removed from consideration more than two thousand Flickr images which were not labeled with any crew and/or any location. Images that did not actually show a crew member, even if one or more names were mentioned in the respective text descriptions (such as an image of an experiment that showed only the relevant equipment, but not the astronaut working on it), were also excluded. This process left 6,262 usable images. Metadata scraping of their descriptions yielded 10,346 identifications of 217 people in twelve modules (one Japanese, one European, six American, and four Russian). One might expect these identifications to be evenly distributed across the modules, whether by gender, nationality, or space agency affiliation, according to the proportions of each group in the overall population of ISS inhabitants and visitors. We show below that this hypothesis is rejected for each of the three variables $(p<.001)$, although the conclusions to be drawn from these results will necessarily be tentative, pending future research. This p-value is for an analysis of variance (ANOVA) test carried out to check if the means of counts of astronauts per gender, nationality, and space agency affiliation are significantly different from each other. The null hypothesis suggests that groups can be considered as part of a larger set of population. For each affiliation, we get a p-value $<0.001$ and thus reject the null hypothesis for all cases.

Figure 4 shows the distribution of the images by module; roughly $75 \%$ of the images show the US Orbital Segment, while 25\% show the Russian Orbital Segment. 


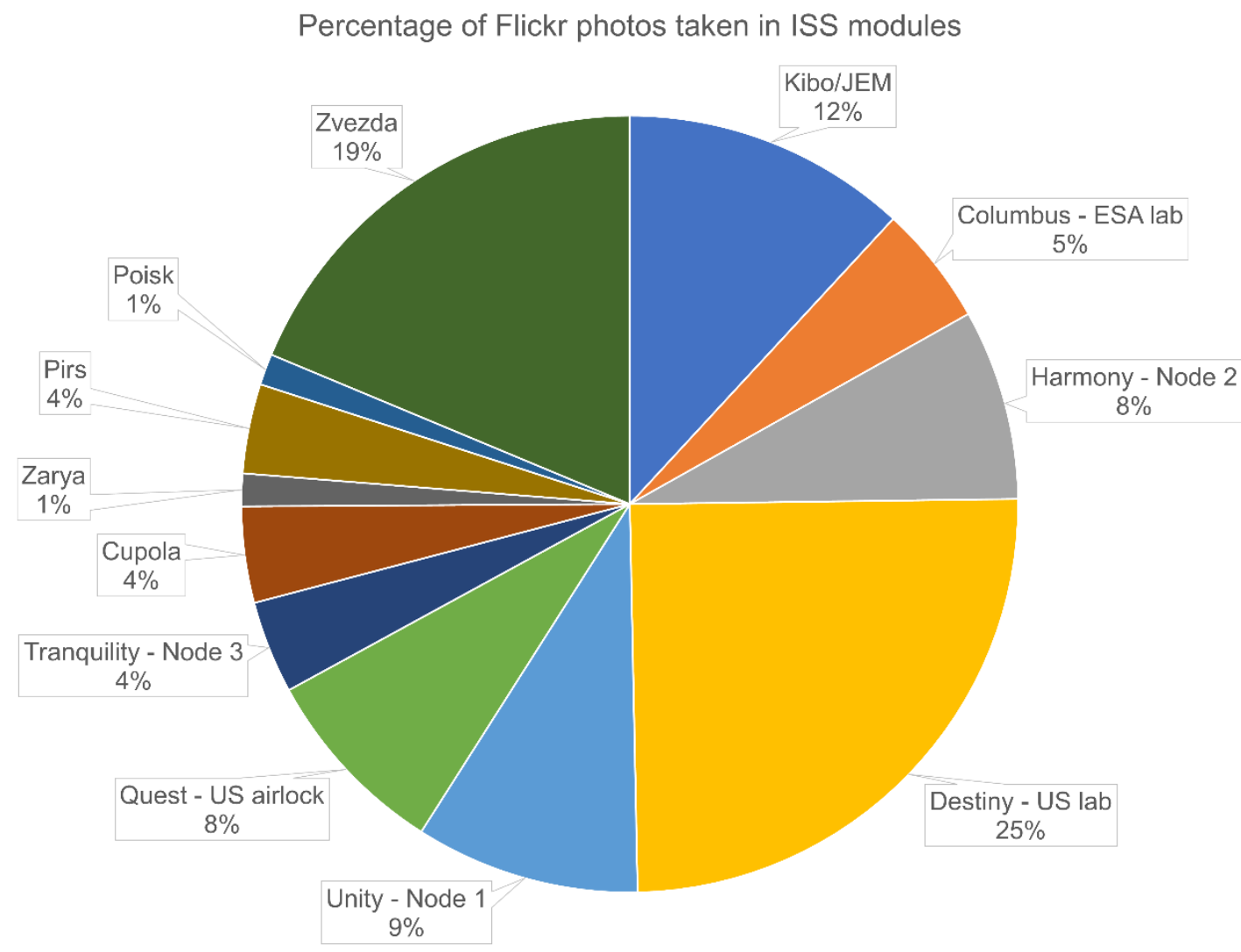

Fig. 4. Percentage of photographs published by NASA on its Flickr site by module shown.

\section{Analysis}

\section{A. Gender in ISS modules}




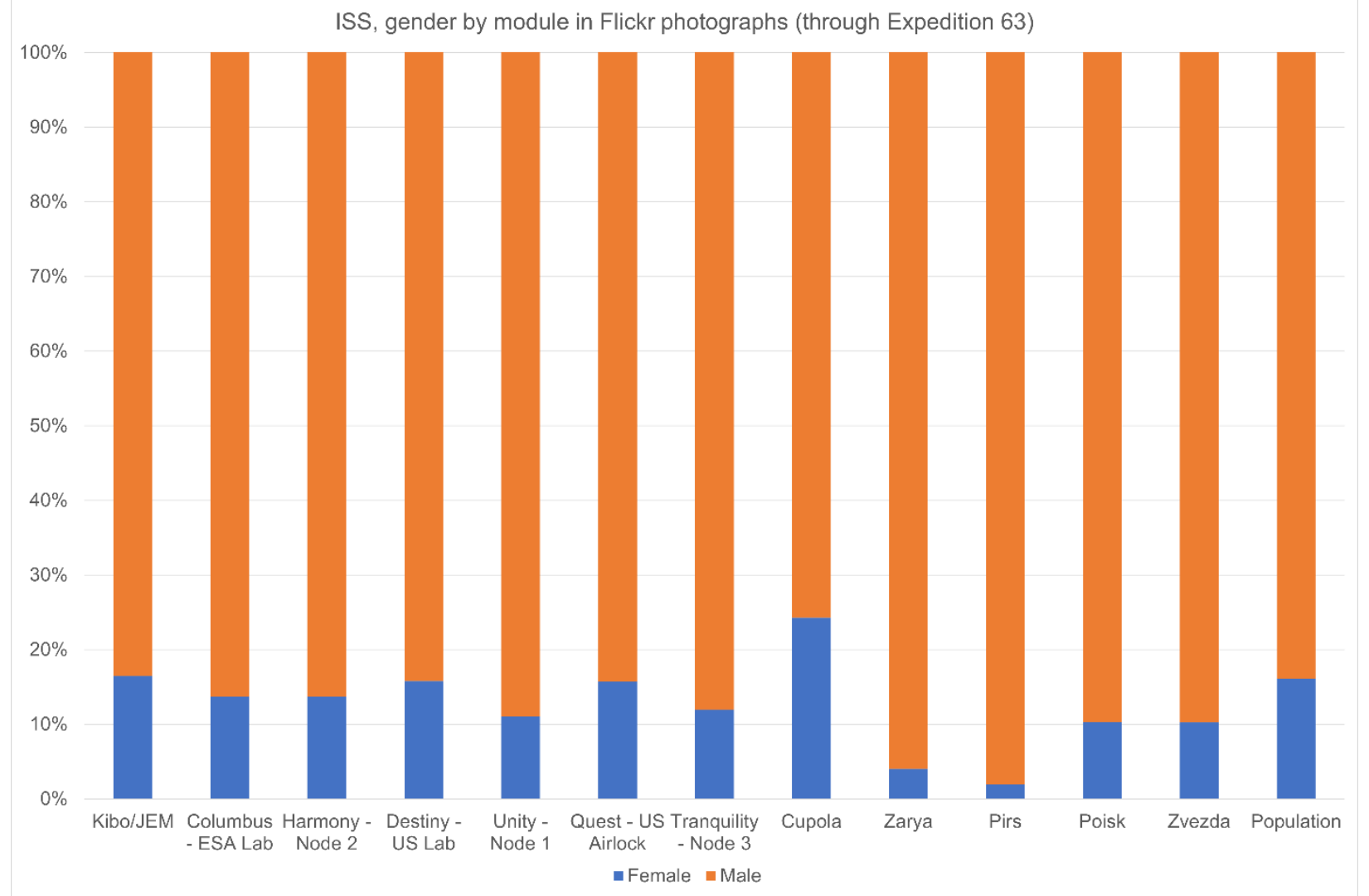

Fig. 5. Proportions of men and women pictured in photos of ISS published by NASA on its Flickr page, organized by module. The bars in each graph are organized from left to right roughly from the front of the space station to the aft, with the US Orbital Segment (consisting of the US, Japanese, and European modules) "ending" at the Cupola, and the Russian Orbital Segment "beginning” at Zarya. For reference, the proportional groupings for the two groups in the overall ISS population are seen in the final bar.

In the first 63 Expeditions, 204 men and 38 women visited ISS. Of these, 32 women were American. In this period, Russia sent one female cosmonaut to ISS, the same as Japan, Italy, France, Canada, and South Korea. In the Flickr images, males comprised $83.9 \%$ of the 217 people captured in the photographs, while females comprised $16.1 \%$ (almost the same as the actual proportion of women in the ISS population, which was $15.7 \%$ ). The distribution of men and women in each module can be seen in Fig. 5. Low percentages of women were most noticeable in the Russian modules Pirs (1.9\%), Zarya (4.0\%), Zvezda (10.2\%), and Poisk (10.3\%). Other slightly low proportions of women were seen Node 1 
(11.1\%), Node 3 (12.0\%), Node 2 (13.7\%), and the European lab Columbus (13.7\%). Slightly higher proportions, close to the actual population, appeared in the Quest airlock (15.7\%) and Destiny lab $(15.8 \%)$. The only areas where women were seen in larger numbers than their proportion of the population would suggest are the Japanese lab module known as Kibo (16.5\%) and the Cupola (24.3\%). The extremely high proportion of women seen in Flickr images of the Cupola (where $n=367$ individuals) seems worthy of note - the proportion of women in this location is $51 \%$ higher than in the general population. The Cupola is often featured as an aesthetically pleasing location for ISS images, one where work is not shown as often as leisure (fig.6). Astronauts are frequently seen simply regarding Earth through its windows.

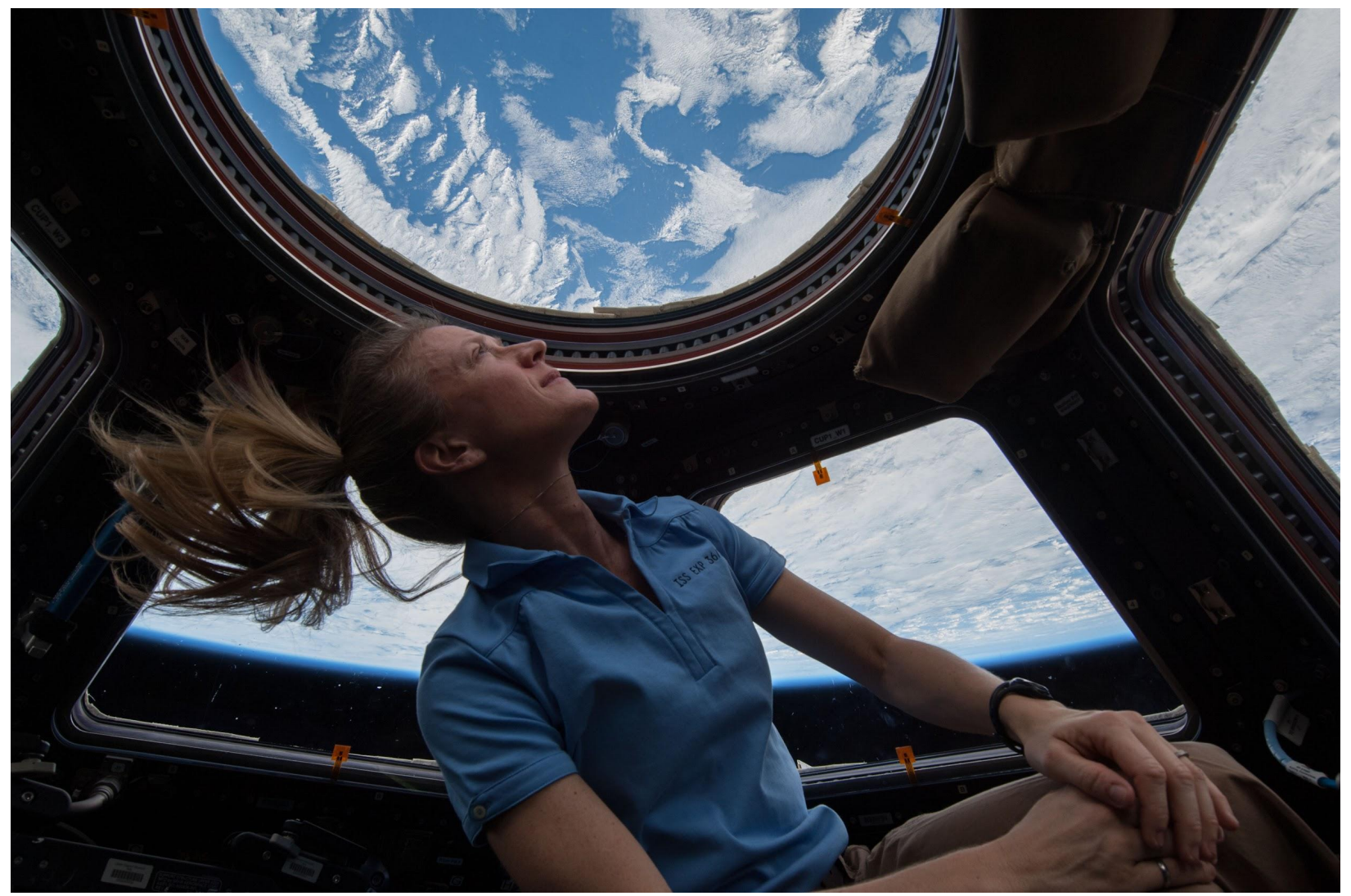

Fig. 6. NASA astronaut Karen Nyberg looks out of the windows of the Cupola at Earth on November 4, 2013. (NASA, by permission)

The emphasis on women in this location in the Flickr photoset may reflect a choice - conscious or unconscious - by NASA's Public Affairs Office to link beautiful images of Earth from space with 
femininity. By contrast, women are seen less frequently in spaces associated with rest, eating, and exercise, and slightly less frequently than average in spaces associated with scientific experimentation. Given the growth of concern about the gender imbalance in the space industry globally, a finer-grained analysis of gendered experiences of the Cupola and other spaces seems likely to produce some useful insights.

The images taken in the Cupola bear some thematic resemblance to representations of women in visual art at the turn of the nineteenth century, as documented by Dijkstra [16]. Paintings and sculptures exhibited in European galleries, and disseminated in prints to the general population, shared common suites of symbols capturing perceptions of femininity. Typically, these included female bodies in passive poses, appearing as if weightless, and in association with mirrors or circles. The persistence of these tropes into such a different twenty-first century context bears further analysis; but may be just as potent in shaping representations of women in space.

\section{B. Nationality in ISS modules}


ISS, nationality by module in Flickr photographs (through Expedition 63)

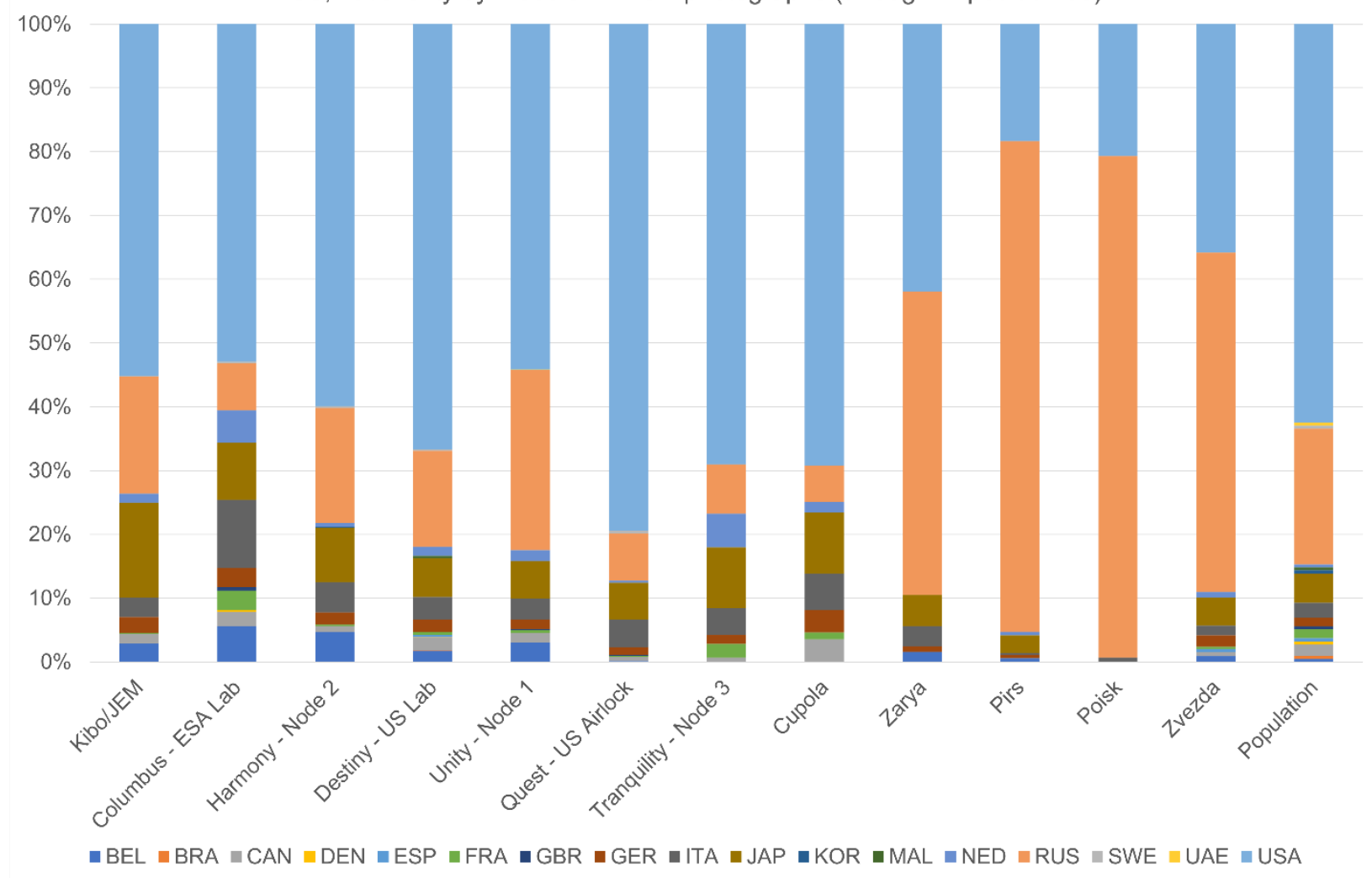

Fig. 7. Proportions of people of different nationalities pictured in photos of ISS published by NASA on its

Flickr page, organized by module.

ISS visitors and inhabitants from seventeen countries appear in the Flickr image set (fig. 7). Of these, Belgium, Brazil, Denmark, Spain, South Korea, Malaysia, Sweden, and the United Arab Emirates are each represented by one individual $(0.5 \%$ each). There were also three Germans $(1.4 \%)$, four Canadians (1.8\%), four French (1.8\%), five Italians (2.3\%), ten Japanese (4.6\%), 46 Russians (21.2\%), and 135 Americans (62.2\%). Beginning with the largest group, there were three locations where Americans were seen more often than their numbers alone would suggest: Node $3(69.0 \%)$, the Cupola (69.2\%), and the Quest airlock (79.4\%). Americans only appeared in numbers roughly proportional to their overall representation in Node 2 (59.2\%) and the Destiny lab (66.7\%). Slightly fewer Americans were seen in Node $1(54.2 \%)$, Columbus (53.0\%), and Kibo (55.1\%); significantly fewer were seen in the Russian Orbital Segment: Zarya (41.9\%), Zvezda (35.7\%), Poisk (20.7\%), and Pirs (18.3\%). These results are almost reversed for the Russian contingent: more were seen in Node 1 (28.2\%), Zarya (47.6\%), 
Zvezda (53.2\%), Pirs (76.9\%), and Poisk (78.6\%). Russians appeared approximately in accordance with their overall proportion in Node 2 (18.1\%) and Kibo (18.4\%), while fewer Russians were seen in the Cupola (5.7\%), Columbus (7.4\%), Quest (7.4\%), and Node $3(7.8 \%)$.

Japanese crew appeared in large numbers in Kibo (14.9\%), the Cupola (9.5\%), Node 3 (9.5\%), and Columbus (8.9\%). Notably, Pirs (2.8\%) and Poisk ( $0 \%)$ were the only modules where Japanese astronauts did not appear in proportions at least equivalent to their actual population. This seems to indicate that Japanese crew appear more frequently in photographs than other inhabitants of ISS. Indeed, the number of images for long-term ISS inhabitants shows that on average, Japanese crew members appear in $16 \%$ more images per day than the average for all crew ( 0.51 compared to 0.44$)$. Italians make up a much larger proportion of the occupants of Columbus (10.7\%) than their population would indicate; they also made up relatively large proportions of people seen in the Cupola (5.7\%), Quest (4.3\%), and Node 3 (4.2\%). Italians were rarely seen in the Russian Orbital Segment, except for Zarya (3.2\%).

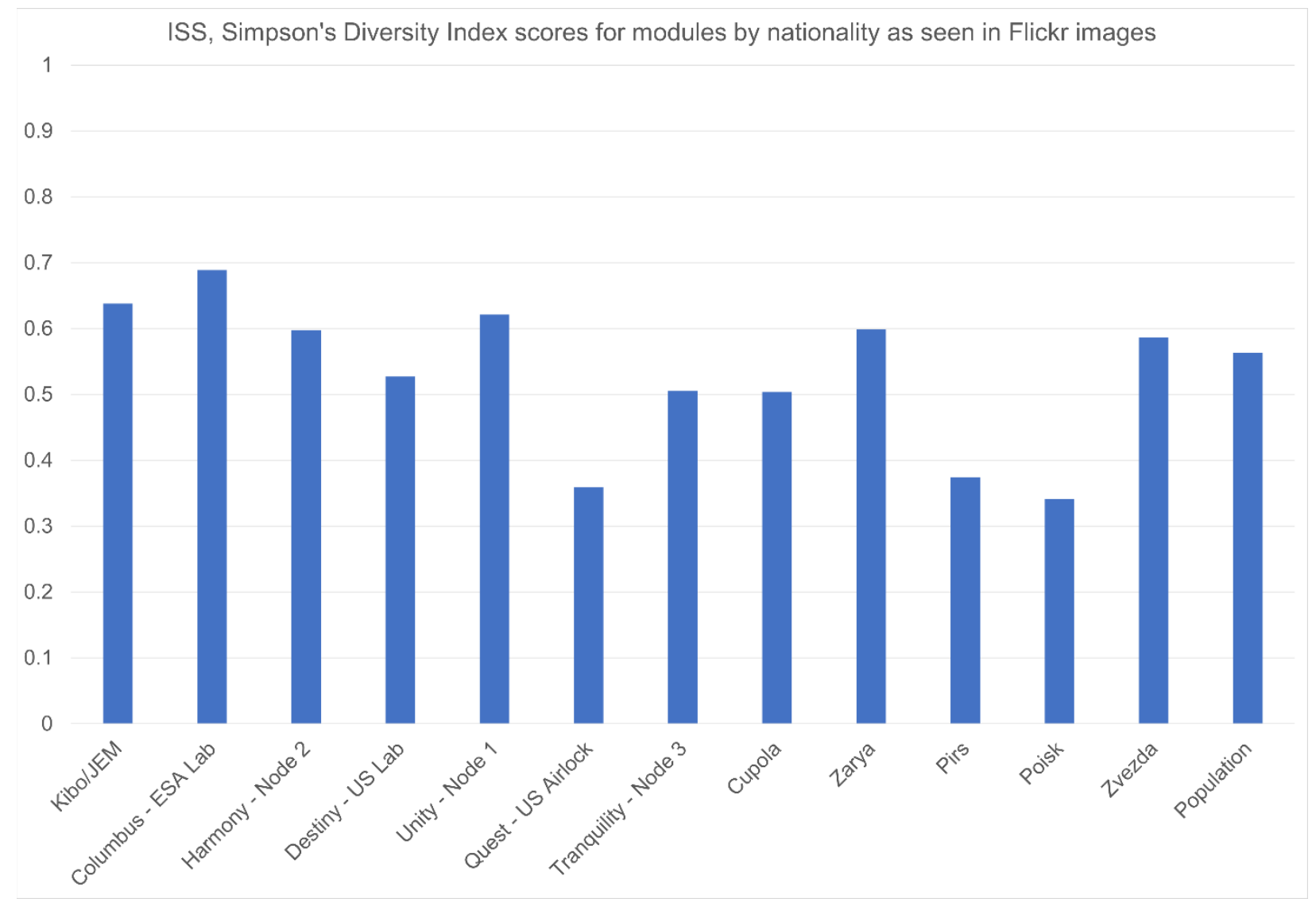


Fig. 8. Simpson's Diversity Index scores for ISS modules by nationality.

It is possible to characterize modules as more or less "international" by reference to a statistical test known as Simpson's Diversity Index (SDI). This test was originally designed for ecology studies, and measured habitat diversity as a function of the number of different species of animals present in a habitat as well as the number of individuals in each species [17]. It can be seen in Eq. (1).

$$
S D I=1-\frac{\Sigma n(n-1)}{N(N-1)}
$$

\section{Eq. 1. The formula for Simpson's Diversity Index.}

In this equation, $n$ is the number of individuals of each species and $N$ is the total number of individuals of all species. A habitat is considered maximally diverse ( $\mathrm{SDI}=1)$ when the number of individuals is evenly distributed across all species. On ISS, this would mean that the population of a module is maximally diverse if whatever nationalities are represented there each had the same number of people. A low diversity score is achieved when one or a few groups are represented by many more individuals than other groups. In these terms, Columbus (0.69), Kibo (0.64), Node 1 (0.62), Node 2 (0.60), and Zarya (0.60) were the most diverse spaces (fig. 8) - a significant result showing that geographic location within the station is not the only determining factor for diverse occupancy. It might be expected that the Cupola would attract occupants from all national groups - but in the Flickr images, at least, fewer than half of all nations are represented there, and the American population of this area is more than twice as large as all other countries combined. Again, this may reflect an effect created by the priorities of the images' publisher, NASA's Public Affairs Office, to show their own crew in images selected for publication. Pirs, Poisk, and Quest were the least diverse, and thus the least international spaces on ISS. The latter two spaces are where extravehicular activity is carried out - an activity that is particularly associated with Roscosmos and NASA, respectively, and which relies on their equipment, which is mutually incompatible. Russians rarely receive training in the US spacesuits, and vice-versa (although European 
and Japanese crew sometimes receive training in both). It is worth noting that Japanese crew make up almost three times as great a proportion of the inhabitants of Kibo as would be expected from their numbers; Italians make up four times as many of the inhabitants of Columbus; and Russians make up more than 3.6 times the populations of Pirs and Poisk. These statistics indicate how, even in an ostensibly international space habitat, national linkages to specific spaces are still strongly apparent.

\section{Space agency affiliation in ISS modules}

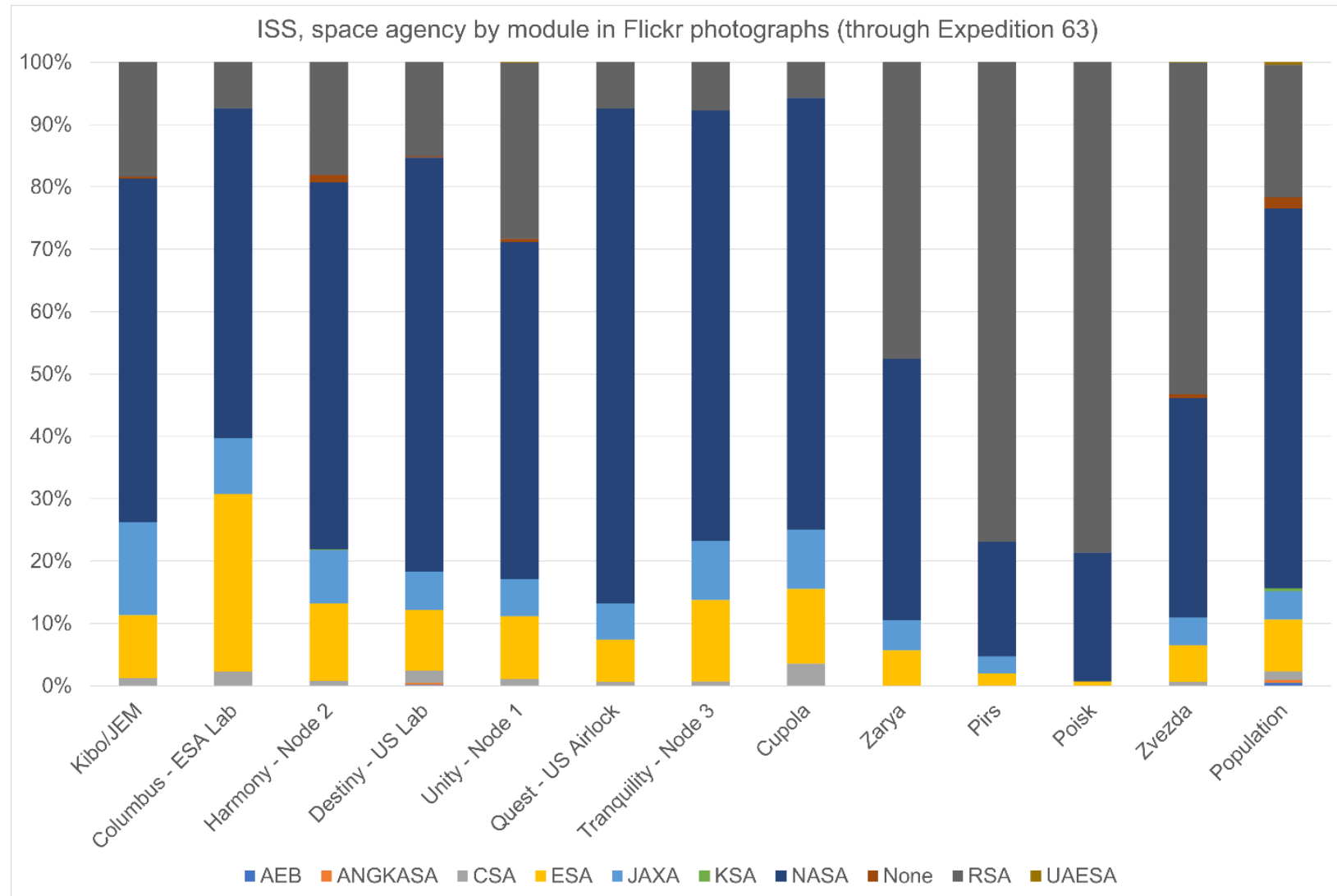

Fig. 9. Proportions of members of different space agencies pictured in photos of ISS published by NASA on its Flickr page, organized by module.

Examination of space agency affiliations of individuals seen in various modules demonstrates, in grosso modo, similar patterns as the study of their nationalities (fig. 9). There are two primary differences, however: first, the crew from nine countries all belong to ESA (18 individuals, $8.3 \%$ ); second, four individuals in the photos (1.8\%) are private astronauts, better known as "spaceflight participants" or 
"space tourists," who do not belong to a space agency, but were included with their respective nations of citizenship in the previous analysis. Four astronauts $(0.5 \%$ each) are unique representatives of the space agencies of Brazil (AEB), Malaysia (ANGKASA), South Korea (KSA), and the United Arab Emirates (UAESA). The other international partner agencies which combine to run ISS are NASA (132 individuals in this photo set, $60.3 \%$ ), Roscosmos (46 individuals, $21.2 \%$ ), JAXA (10 individuals, 4.6\%), and CSA (3 individuals, $1.4 \%$ ). In this discussion, we will concentrate on the European contingent, since the other space agencies map almost directly onto the national distributions previously described. In the US Orbital Segment, ESA crew made up a larger proportion of crew population than would be expected in every module except Quest (6.8\%). In Columbus, the European lab module, they made up $28.5 \%$ of the population, more than triple the actual population. Node $3(13.0 \%)$, Node $2(12.5 \%)$, and the Cupola $(12.0 \%)$ were also notably high. Private astronauts make up only a very small percentage of the population of any USOS module, reaching $1 \%$ only in Node 2 . This fact may be due to NASA's general disapproval of their presence on ISS in the first decade of the station's use. It will be interesting to see whether private astronauts become more visible in published photographs as NASA opens up new opportunities for their visits in 2022. 


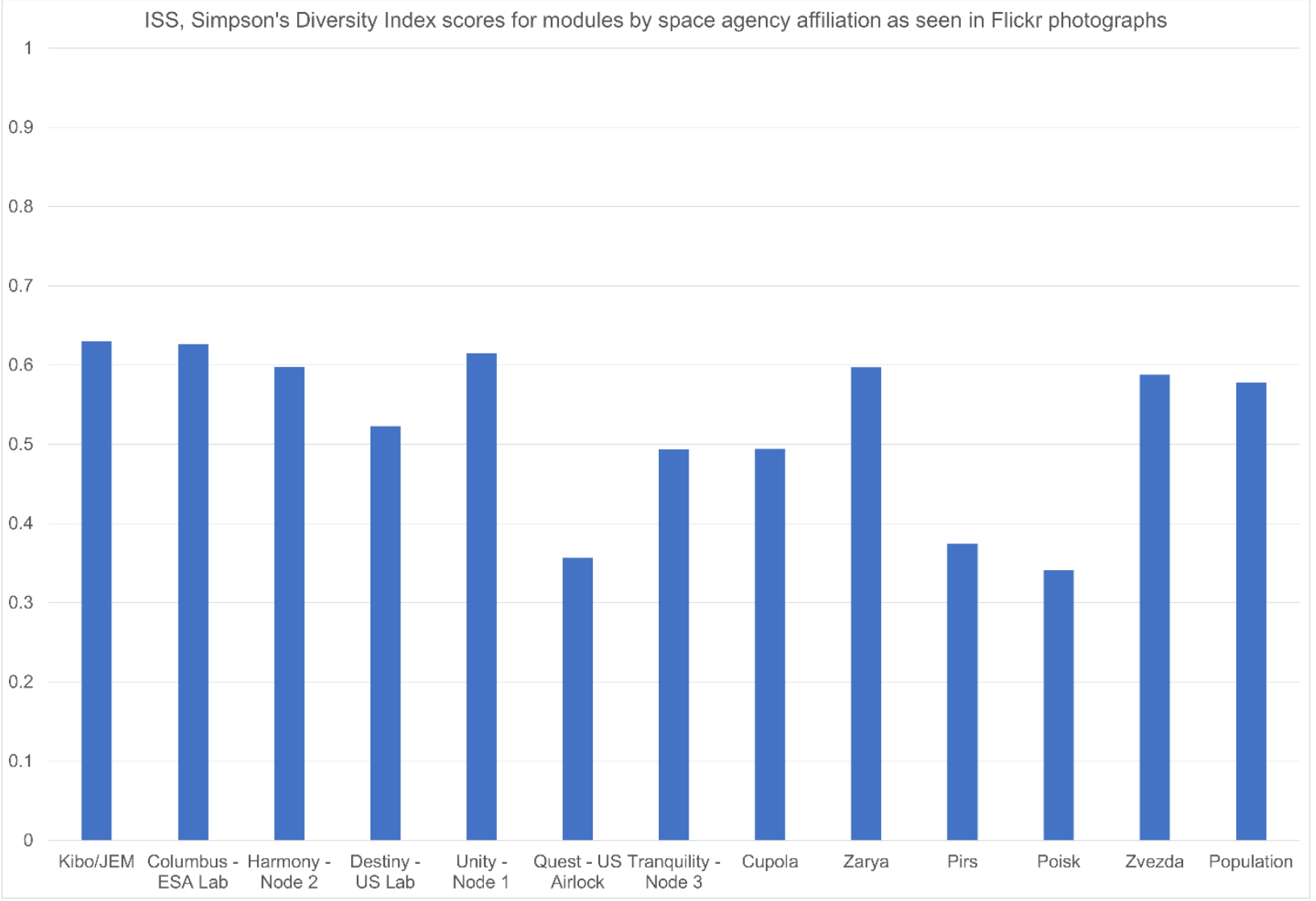

Fig. 10. Simpson's Diversity Index scores for ISS modules by space agency affiliation.

With regard to diversity among the space agencies, as measured by Simpson's Diversity Index, the smaller number of groups containing the same total number of individuals yielded a more diverse distribution overall, although still not reaching high diversity levels of 0.8 or more (fig. 10). Most of the modules, including Kibo, Columbus, Node 2, Node 1, Zarya, and Zvezda, were at or close to 0.6. Only the two airlocks, Quest (0.36) and Poisk (0.34), and the BEAM (0.41) and Pirs (0.37) modules showed somewhat lower diversity.

\section{Biases in the data}

It is difficult to define with precision the difference between what is shown in the images and what the actual distribution of people across the station has been at any given moment. We must note three identifiable sources of bias in the set of photographs used for this study. First is the fact that the 
Flickr images do not include images held in the archives of the Roscosmos State Corporation for Space Activities or its primary contractor, RSC Energia. Such images have the potential to reveal different relative distributions of people, especially in the Russian Orbital Segment (ROS). We can say with certainty that the percentage of images in the Flickr set which show the ROS have declined precipitously over time, beginning already in 2001 (Fig. 11). Since 2011, after NASA added its own crew berths to Harmony - Node 2, the percentage of published photos showing the Russian part of ISS has been below $20 \%$ for all but one year, and it has been below $10 \%$ four times. We hope in the future to gain access to the Russian images to eliminate this source of bias, but the announcement in 2021 of new government rules governing the sharing of data about the Russian space industry with foreigners seems to make this an unlikely prospect.

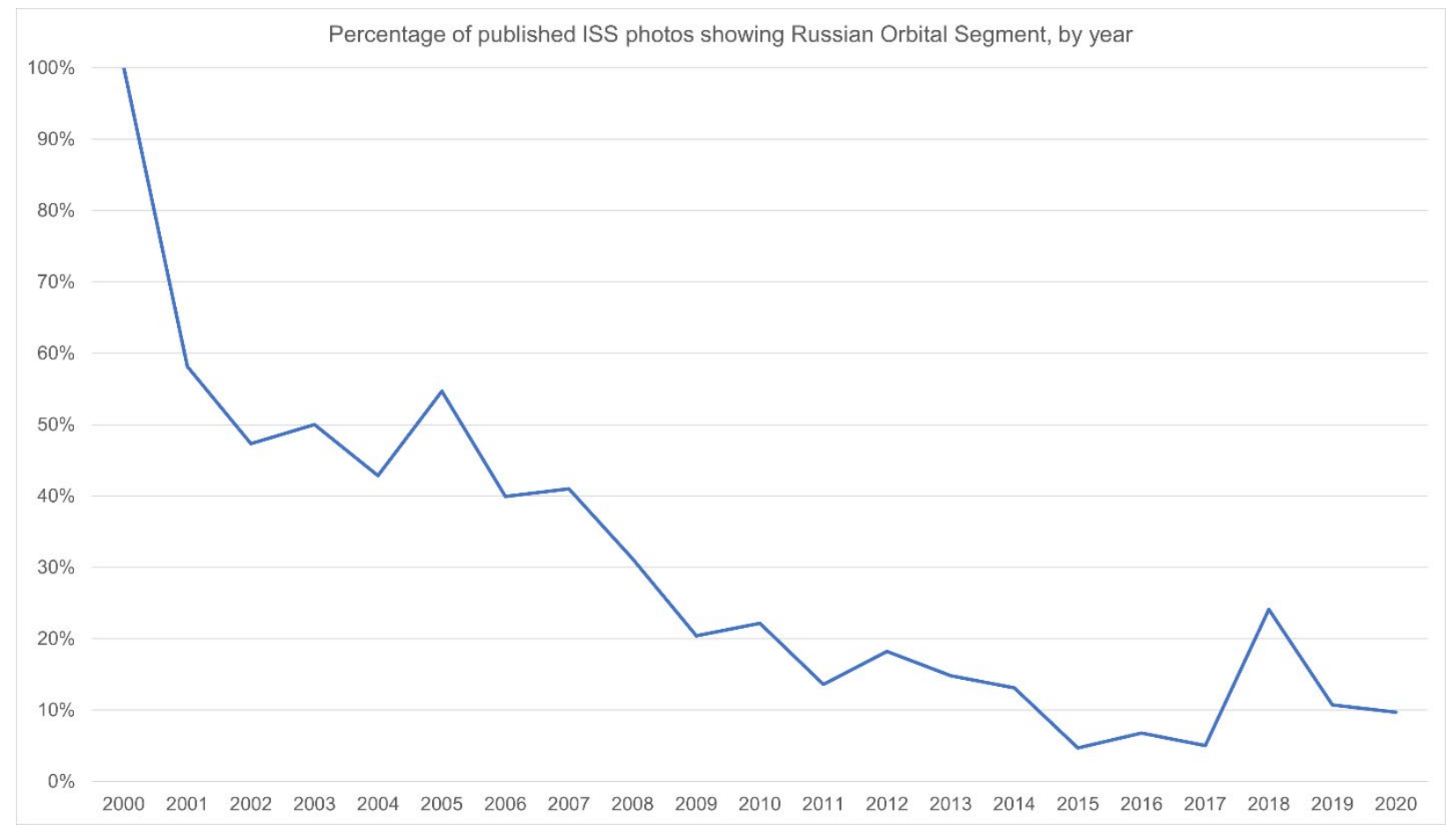

Fig. 11. Percentage of photographs appearing on NASA's Flickr site which show modules in the Russian Orbital Segment of ISS, by year.

One other area of bias related to the chronological distribution of the images is that the site was extended spatially over time as the station was built out and new modules were added. ISS was not considered essentially complete until the permanent addition of the Permanent Multipurpose Module 
known as Leonardo in March 2011. Leonardo is used almost exclusively for storage, and was only shown in eight published photos, so its results are not included here.

Even now, more modules are expected to be added in coming years, such as the Russian Nauka (Science) module, which arrived in July 2021. A somewhat mitigating factor to this bias is the small increase in the number of images in the last decade relative to earlier (2000-2010, average 312 pictures per year; 2011-2020, average 371 per year; fig. 12), although the bulk of the photographs are clustered in the first half of the decade.

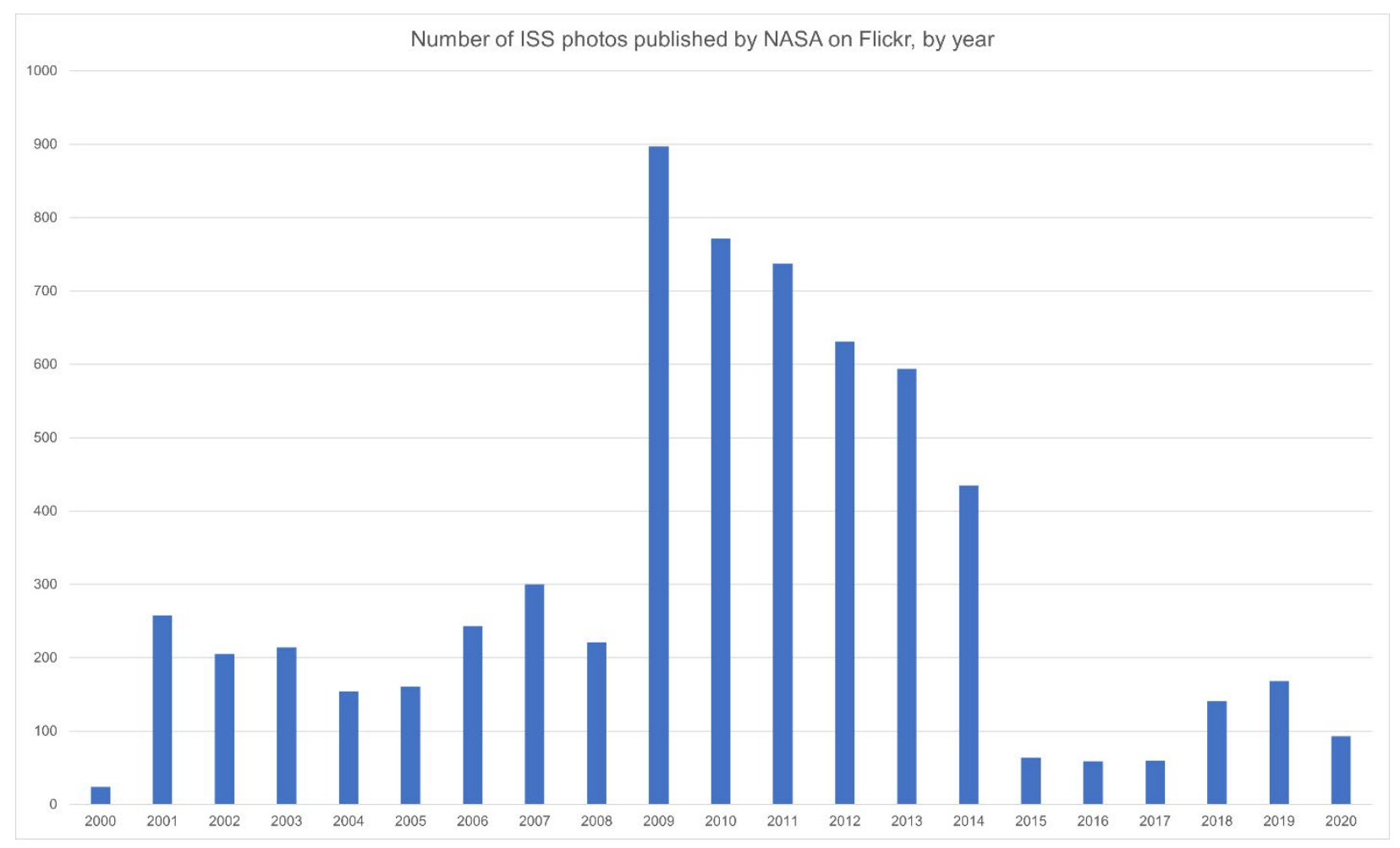

Fig. 12. Count of ISS pictures published by NASA on its Flickr site, by year.

A final source of bias originates from the fact that the Flickr images were selected for publication from a larger set that are maintained by one agency, NASA. The curation of the Flickr images was done by staff of NASA's Public Affairs Office (PAO), according to its priorities and interests, presumably focusing on the promotion of a positive public view of NASA and the ISS project. Indeed, one possible interpretation of the patterns identified above could be that, rather than demonstrating evidence of actual population distributions, we have instead only discovered that they reveal the curatorship of the images by 
NASA. We have already pointed out one way that the PAO's priorities might be visible, in the ways women are shown in substantially greater numbers in the Cupola. At the same time, however, many aspects of the patterns clearly reflect outcomes that could be expected based on prior understandings of ISS political structures, such as the differences in populations between the US and Russian Orbital Segments. Future work on the full set of images (now being made available to us) will help to illuminate the effect of this source of bias. Pending funding, approval by the international partner agencies, and the consent of participating crew members, we also hope to implement a third phase of this study using a completely different data set: direct monitoring of individuals' movements around the station using astronaut-worn RFID tags. Each crew member will keep an anonymized tag on their person or in their clothing for a period of one or more weeks. As they move from module to module, the tags will be recorded by readers, allowing us to understand how much time different people spend in different locations. Comparing this systematic short-term study to the longer-term but more random data from the photographs should give a much clearer view of actual population distributions.

\section{Conclusion}

Even though this analysis is relatively basic - it can be encapsulated by the simple question, "who was where on ISS?" - it is still unique: no space agency has ever been able to establish raw data for the gendered or national groups living in an orbital habitat. These results begin to fulfill the promise of archaeological approaches to human interactions with the material culture of space. They demonstrate relevance to future mission planning and habitat design. We will gain an even better understanding of population distributions in the future by examining the full set of NASA photographs (and hopefully the Russian ones, as well) and by integrating information about objects and activities into our study. Further ML analysis of other factors correlating with the categories used here may illuminate causes of the observed distributions other than agency, nationality and gendered affiliations. 
Even in this preliminary study, the analysis of people in the images seems to indicate the challenges of creating a space habitat whose population distribution truly reflects its internationalist ideals. The tendency of Russians to appear in their own modules, but not elsewhere, and the concomitant tendency of other groups (including women as a distinct class) to appear in the US, European, and Japanese modules, but not in the Russian ones, shows that the space station may fairly be said to be divided down the middle at the Pressurized Mating Adapter that links Node 1 and Zarya. This fact is perhaps less surprising if we consider that the agreements that govern the International Space Station project for all practical purposes did, from the outset, divide responsibilities and jurisdictions for the station into two clearly delineated zones: the US and Russian Orbital Segments [18, 19, 20]. For all that ISS is a cooperative multi-agency project, it is well-known that experiments - the primary work of the crew, other than constructing and maintaining the station - are not managed by a single joint oversight board, but instead by each individual agency according to its own priorities, and then placed on board as payloads via negotiation with the others. The crew are therefore not precisely a unified team, even though they do some training together before launch and work on some tasks together. Further, the various facilities on the station are explicitly labeled as having agency affiliations - as belonging to one or another nation or group of nations.

We can tentatively suggest that due to the way responsibilities, jurisdiction, and work have been divided on ISS, the station likely falls short of optimal efficiency with regard to its human resources. Future studies may show that the imbalanced population distributions we have identified are actually detrimental to overall crew cohesiveness and morale (see, for example, NASA astronaut Chris Cassidy's recent discussion of his loneliness during the time when he was the only American - and only resident of the US Orbital Segment - on board, together with a pair of cosmonauts, during Expedition 63) [21]. These issues should be considered serious matters of concern for a project that is one of the most expensive building projects ever undertaken by humanity. Indeed, habitat design must be a key research area in NASA's Human Research Program. As Landon et al. state in their study Risk of Performance and Behavioral Health Decrements Due to Inadequate Cooperation, Coordination, Communication, and 
Psychosocial Adaptation within a Team, "habitat design calls for adequate volume and layout supporting team activities (e.g., training, social time, community meals) and cohesion” [22, 23]. New cooperative space habitat projects such as the NASA-ESA-JAXA-CSA Lunar Gateway orbital station and the proposed Russian and Chinese joint lunar base should consider how to create unified control structures such as a single oversight board to determine what work will be done, composed of members from all participating groups, and architectures that are fully shared by crew regardless of their gender, national origin, or space agency affiliation.

\section{References}

[1] S.M.Fuller, M.R. Davis, and H.J. Durham. "Paper session I-A - planning for operations on-board the International Space Station.” The Space Congress ${ }^{\circledR}$ Proceedings [Internet]. 1997;6. Available from: https://commons.erau.edu/space-congress-proceedings/proceedings-1997-34th/april-29-1997/6

[2] R. Leuttgens and. J. Volpp. “Operations planning for the International Space Station.” ESA Bulletin 1998;94. European Space Agency. Available from:

https://www.esa.int/esapub/bulletin/bullet94/LUETTGENS.pdf

[3] J.J. Marquez, S. Hillenius, M. Healy, and J. Silva-Martinez. "Lessons learned from International Space Station crew autonomous scheduling test.” [Internet] 2019; Available from:

https://ntrs.nasa.gov/api/citations/20190027148/downloads/20190027148.pdf?attachment=true.

[4] J. Walsh and A.C. Gorman. "A method for space archaeology research: the International Space Station Archaeological Project.” Antiquity 2021 Oct;95(383): 1331-1343. doi:10.15184/aqy.2021.114

[5] NASA. "NASA Johnson.” 2021; Available from: https://www.flickr.com/photos/nasa2explore/

[6] W. .Salmond, J. Walsh, and A. Gorman. "Eternity in Low Earth Orbit: Icons on the International Space Station.” Religions 2020;11(11): 611. https://doi.org/10.3390/rel11110611 
[7] J. Walsh, A.C. Gorman, and W. Salmond. "Visual Displays in Space-Station Culture." Current Anthropology 2021 Dec; 62(6).

[8] M. Hedges, and S. Dunn. Academic Crowd-Sourcing in the Humanities. London: Chandos Publishing; 2018. https://doi.org/10.1016/C2015-0-04363-5

[9] A. Katiyar, V. Srividya, and B. K. Tripathy. "TagIT: A System for Image Auto-tagging and Clustering." Data Engineering and Intelligent Computing. Singapore: Springer; 2021. p. 259-268.

[10] C. Lintott, The Crowd and the Cosmos. Oxford:Oxford UP; 2019.

[11] A. Kittur, E.H. Chi, and B. Suh. "Crowdsourcing user studies with Mechanical Turk." Proceedings of the SIGCHI Conference on Human Factors in Computing Systems. New York: Association for Computing Machinery; 2008. p. 453-456. https://doi.org/10.1145/1357054.1357127

[12] K. Kyriakou, P. Barlas, S. Kleanthous, and J. Otterbacher. "Fairness in proprietary image tagging algorithms: A cross-platform audit on people images." Proceedings of the International AAAI Conference on Web and Social Media 2019;13(1): 313-322.

[13] B. Mozafari, P. Sarkar, M. Franklin, M. Jordan, and S. Madden. 2014. "Scaling up crowd-sourcing to very large datasets: a case for active learning." Proceedings of the VLDB Endowment 8 (2): 125-136. https://doi.org/10.14778/2735471.2735474.

[14] NASA Johnson. “Astronauts Jessica Meir and Andrew Morgan work on orbital plumbing tasks.” iss061e144870, 2020 Jan 26. Available from: https://www.flickr.com/photos/nasa2explore/49464133033/in/album-72157704730697552/.

[15] D. Glez-Peña, A. Lourenço, H. López-Fernández, M. Reboiro-Jato, F. Fernández-Riverola. Briefings in Bioinformatics 2014;15(5): 788-797. https://doi.org/10.1093/bib/bbt026. 
[16] B. Dijkstra. Idols of Perversity: Fantasies of Feminine Evil in Fin-de-Siècle Culture. Oxford: Oxford UP: 1987.

[17] E.H.Simpson. "Measurement of Diversity." Nature 1949;163:688.

[18] United States Department of State. "Russian Federation (96-611) - Protocol Regarding the Balance of Their Contributions to the International Space Station.” 1996 June 11. Available from: https://www.state.gov/96-611/.

[19] NASA-RSA. "NASA-RSA Agreement: memorandum of understanding between the National Aeronautics And Space Administration of the United States Of America and the Russian Space Agency concerning cooperation on the civil International Space Station.” 1998 Jan 29. Available from: https://www.nasa.gov/mission_pages/station/structure/elements/nasa_rsa.html.

[20] D. St.-Arnaud., A. Farand, , M. Uchitomi, R.J. Frank, and I. Porokhin.. "The legal framework for the International Space Station.” United Nations Committee on the Peaceful Uses of Outer Space Legal Subcommittee, 2013 Apr 17. Available from: https:/www.unoosa.org/pdf/pres/lsc2013/tech-05E.pdf.

[21] C. Thorp. "What it's like to be alone on the International Space Station." InsideHook [Internet]. 2021

Oct 7. Available from: https://www.insidehook.com/article/science/nasa-chris-cassidy-aloneinternational-space-station.

[22] L. Blackwell Landon. W.B. Vessey, and J.D. Barrett. "Evidence report: risk of performance and behavioral health decrements due to inadequate cooperation, coordination, communication, and psychosocial adaptation within a team.” NASA Human Research Program; 2016. Available from: https://humanresearchroadmap.nasa.gov/evidence/reports/team.pdf.

[23] A.R. Kearney. "Team Health and Performance in Spaceflight Habitats:Risks, Countermeasures, and Research Recommendations.”NASA Technical Document, TM-2016-219274. 2016. 